NASA Technical Memorandum 101405

ICOMP-88-21; AIAA-89-0966

\title{
Effects of Core Turbulence on Jet Excitability
}

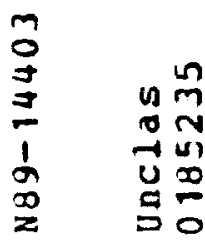

Reda R. Mankbadi

Institute for Computational Mechanics in Propulsion

Lewis Research Center

Cleveland, Ohio

Ganesh Raman

Sverdrup Technology, Inc.

NASA Lewis Research Group

Cleveland, Ohio

and

Edward J. Rice

National Aeronautics and Space Administration

Lewis Research Center

Cleveland, Ohio

Prepared for the

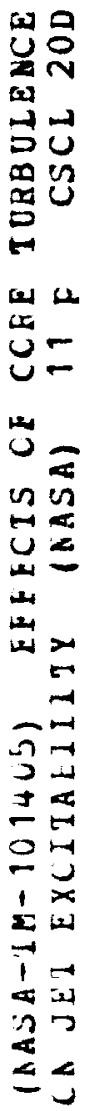

Second Shear Flow Control Conference

sponsored by the American Institute of Aeronautics and Astronautics

Tempe, Arizona, March 13-16, 1989

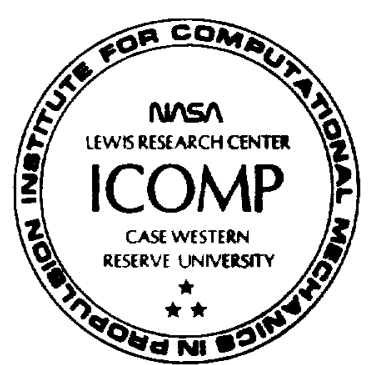





\title{
EFFECTS OF CORE TURBULENCE ON JET EXCITABILITY
}

\author{
Reda R. Markbadi * \\ Institute for Computational Mechanics in Propulsion \\ Lewis Research Center \\ Cleveland, Or io 44135 \\ Ganesh Raman \\ Sverdrup Technology, Inc. \\ NASA Lewis Research Center Group \\ Cleveland, Ohio 44135 \\ and \\ Edward J. Rice \\ National Aeronautics and Space Administration \\ Lewis Research Center \\ Cleveland, Ohio 44135
}

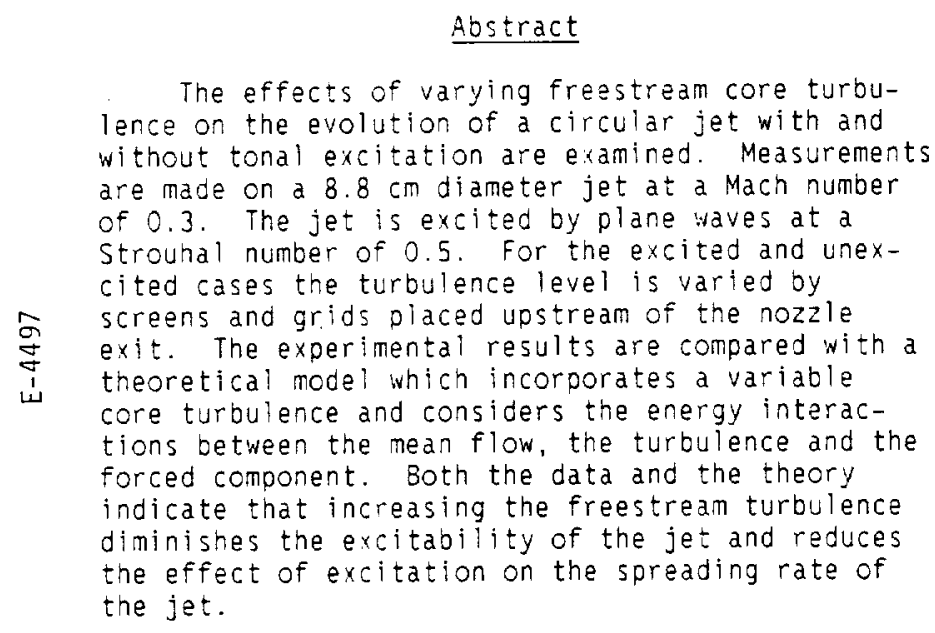

Introduction

Excited jets have been the subject of several recent investigations. $1-3$ These investigations are concerned with the fundamental understanding of the jet development as well as possible technological applications such as control of the jet mixing. The growth of a forced periodic velocity component is governed by several parameters. Zaman \& Hussain 4 considered the effect of excitation on Strouhal number as well as the excitation level on the development of the periodic flow components. The effect of the initial velocity profile was considered by Chan and Templin. 5

In order to make flow control techniques technologically attractive it has to be demonstrated that this "control" can be exerted in "real life flow "which unlike the laboratory flows has higher leveis of freestream turbulence. It is al so of fundamental interest to understand how the initial turbulence controls the growth of stability waves in excited shear layers. The present work is therefore concerned with examining the effect of the initial turbulence on the development of excited jets.

* Work funded under Space Act Agreement C99066G. On leave frm Cairo University, Cairo, Egypt.
The initial turbulence profile is governed by two parameters: the boundary layer turbulence and the core turbulence. Attention is focused here on the core turbulence, keeping the initial level of the boundary layer turbulence as constant as possible.

The development of a single frequency component in a turbulent round jet was theoretically investigated by Mankbadi \& Liu. 5 This theory is modified here to account for variable core turbulence. On the experimental side, the effect of initial turbulence was examined by Raman, Zaman and Rice. ${ }^{7}$ Various levels of turbulence were created at the nozzle exit by specially fabricated grids placed upstream of the jet exit. Measurements were made of the mean and fluctuating quantities. Their data are compared here with the modified theory. The computations not oniy provide a theory versus experiment comparison but also help in obtaining a better physical picture of the phenomenon.

\section{Experimental Apparatus and Procedure}

The jet facility as presented in Ref. 7 consisted of a $76 \mathrm{~cm}$ diameter plenum chamier supplied with pressurized air. The flow passed through three sireens and two stages of contraction before exiting through the $8.8 \mathrm{~cm}$ diameter nozzle. A $20.3 \mathrm{~cm}$ butterfly valve bypassed by a $3.8 \mathrm{~cm}$ Annin plug valve, both operated remotely, were used to control the flow. A $41 \mathrm{~cm}$ diameter section located between the two contracting sections housed the excitation system. This consisted of four acoustic drivers that were equally spaced around the circumference. Each driver was enclosed in a sealed can and vented to equalized pressure across the driver diaphragm.

Figure 1 is a schematic showing the geometry and location of the turbulence generating grids in the jet facility. The three shown grids represent the three cases of initial core turbulence considered. The three cases will be labeled here 0.5 , 3 , and 5 percent indicating the initial centerline turbulence as percentage of the exit mean centerline velocity. For all the cases the grid blockage was less than 40 percent. The grids were located $33 \mathrm{~cm}$ uostream of the nozzle exit where the diameter of the contracting section is $13.1 \mathrm{~cm}$; the nozzle ended with a sharo edge and had a $22 \mathrm{~cm}$ long cylindrical section prior to the exit. 
A $0.54 \mathrm{~cm}$ ( $B \& K$ ) microphone with nose cone was used to obtain sound pressure levels and spectra. Measurements of mean and fluctuating velocity were made with single and crossed hot-wires together with constant temperature (DISA and TSI) anemometers. Cross-wire data are not reported here but can be found along with further details of the experiment in Raman, Zaman and Rice. ${ }^{7}$

\section{Theoretical Background}

The theory of Mankbadi \& Liu6 is concerned with the development of a single mode, single frequency component of stabllity waves in a turbulent round jet. In this theory ohase-averaging along with the conventional time-averaging techniques are applied to the full incompressible momentum equations to obtain the governing momentum equations for the mean flow, the stability component and the turbulence component. The kinetic energy equations representing the interactions among the three flow components are obtained from the corresponding momentum equations. These energy equations are integrated across the jet. Shape assumptions are made regarding the radial profile of each flow component. The shape of the mean flow is assumed to follow the two-stage hyperbolic tangent profile of Michalke. 8 The profile of the stability component follows that obtained by the solution of the local linear stability equations. The profile of the turbulent stresses is taken to be Gaussian with a peak at the center of the shear layer. The basic contribution to the theory of the present work is modification of the turbulence's shape assumption. To account for the core turbulence, the assumption of the turbulence profile is now changed to be Gaussian within the shear layer but with a prescribed value of turbulence within the potential core equal to the initial core turbulence.

With these shape assumptions the integrated energy equations take the following form:

Mean flow:

$$
\frac{1}{2} \frac{d I}{d \theta} \frac{d \theta}{d x}=-I_{R S}^{\prime}(\theta) E-\tilde{I}_{R S}(\theta, S t, n)|A|^{2}
$$

Fundamental:

$$
\begin{aligned}
\frac{d}{d x}\left[I_{2}(\theta, S t, n)|A|^{2}\right]= & \tilde{I}_{R S}(\theta, S t, n)|A|^{2} \\
& -I_{W t}(\theta, S t, n)|A|^{2} E
\end{aligned}
$$

Turbulence:

$$
\frac{d}{d x}\left[I_{3}(\theta) E\right]=I_{R S}^{\prime}(\theta) E+I_{W t}(\theta, S t, n)|A|^{2} E-I_{\varepsilon}(\theta) E^{3 / 2}
$$

The initial conditions are: $\theta(0)=\theta_{0}$. $E(0)=E_{0},|A(0)|^{2}=\left|A_{O}\right|^{2}$, where $n$ is the azimuthal wave number which is zero in the present analys is since we only consider axisymmetric waves. With these measured quantities of initial conditions as input, the equations are solved to obtain $|A|^{2}$. $E$ and $\theta$ along the jet.

In the above system of equations, $\theta$ is the momentum thickness of the jet, $E$ is the turbulence energy integrated across a slice of the jet and $A$ is the energy of the fundamental component integrated across a slice of the jet. The integrals appearing in the above system of equations are dependent on the shape assumptions and are defined in Mankbadi \& Liu (1981). I is the mean flow energy advection integral. Equation (1) is actually an equation for the streamwise development of $\theta(x)$ with $d I_{1} / d \theta<0$. Thus, $\theta(x)$ will grow as long as energy is extracted from the mean flow by the fluctuations. IRS is the production integral of the turbulence. $\tilde{I}_{R S}$ is the production integral of the fundamental. $I_{2}$ is the energy advection integral of the fundamental component and $I_{W t}$ is the fundamental-turbulence energy exchange integral. For the range of parameters considered here, IWt is positive indicating a transfer of energy from the fundamental to the turbulence. I 3 is the turbulence energy advection integral and $I_{\varepsilon}$ is its viscous dissipation integral. These integrals have the same definitions as given in Mankbadi \& Liu. $I_{2}$. I 3 , and $I_{\varepsilon}$ depend on the shape assumption of the turbulence. Their definitions are still as in Ref. 6 but their fitted functions are now modified to be dependent on the initial core turbulence as well as the momentum thickness. The solution of the above system of equations is thus not only a function of the initial conditions but also of the initial core turbulence which alters the above mentioned integrals.

\section{Results}

In the following we will present the effect of initial core turbulence on the development of the jet. Three cases of initial core turbulence are considered: $0.5,3$, and 5 percent. The other conditions at the nozzle exit are kept as constant as possible. In the results presented here for the excited jet the excitation strouhal number is 0.5 and the initial axial velocity component of the fundamental component of the excitation at the centerline is 0.4 percent of the jet exit velocity.

The measured longitudinal mean velocity, $U$, and the turbulence intensity, $u^{\prime}$, profiles at the jet exit are shown in Fig. 2. The data have been normalized by the jet exit velocity at the centerline. The turbulence measured in the boundary layer at positive $r$ is nigher because the probe and its support mechanism entered the flow to reach this side; the flow impingement resulted in small probe vibrations which were sufficient to contaminate the data in the thin boundary layer. All subsequent measurements are on the axis or in the boundary layer with negative $r$ where only the probe and the stem entered the flow and the vibrations are negligible. The initial turbulence profiles of Fig. 2 are used to calculate the integrated turbulence energy $E(O)$ which is to be used as one of the boundary conditions for the analysis.

The variation of the shape factor, the momentum thickness and the peak fluctuation level in the shear layer measured at $0.5 \mathrm{~mm}$ downstream of the nozzle lip, are shown in Fig. 3. These data demonstrate that the nozzle exit boundary layer characteristics are practically the same for the different initial turbulence levels. For instance, the momentum thickness varies between $\theta / d=0.007$ to 0.073 when the turbulence varies from 0.59 to 5 percent at Mach number 0.2 . The momentum thickness is taken to be 0.0070 for the analysis of the excited jet at 
the three initial turbulence levels as well as for the unexcited cases.

The effect of initial turbulence on the development of the fundamental component is shown in Fig. 4. The experimental data shown in Fig. 4(a) indicate that increasing the initial level of turbulence dampens the amplification of the fundamental component. The same features are also obtained theoreticaliy as Fig. $4(\mathrm{~b})$ indicates. In the experimental dati the fundamental component is almost constant up to $x / 0=0.5$ then begins to amplify. This is pernaps due to the unsteady pressure field at the nozzle exit introduced by the excitation mechanism. This pressure field suppresses the amplification of the fundamental. Such a mechanism is not present in the theory and therefore the computed results indicate that the fundamental begins to grow immediately after it leaves the nozzle. This is one of the factors that may account for the higher computed amplifications of the fundamental as compared to the observed ones.

In the present theoretical analysis only one instability component is assumed to exist. However, examining the measured spectra shown in Fig. 5 indicates the generation of a subharmonic of the fundamental. By $\times / 0=4$, this subharmonic is almost of the same order as the fundamental. The generation of a subharmonic reduces the amplification of the fundamental as discussed in Mankbadi. 9 since the generation of a subharmonic is not accounted for in the present analysis, the calculated amplification of the fundamental is higher than the measured one. However, the present theory clearly indicates that the ampiification rate of the fundamental is reduced by increasing the initial level of core turbulence as the measurements indicate.

The damping effect of the turbulence on the growth of the fundamental can be explained from the present theory as follows. The growth of the fundamental as given by $\mathrm{Eq}$. (2) is governed by the balance between the production of the fundamental by the mean flow and the energy of the fundamental drained by the turbulence. Increasing the turbulence level controls the growth of the fundamental through two mechanisms. First, a higher turbulence level means higher energy drain from the mean flow (the first term in Eq. (1) is proportional to E). Consequently, less mean flow energy is available for the growth of the fundamental. In the second mechanism, which is the dominant one, the energy absorbed from the fundamental by the turbulence is proportional to $\mathrm{E}$ (the second term in Eq. (2)). Therefore higher turbulence levels lead to higher energy drains from the fundamental. The two mechanisms combined cause the initial turbulence to suppress the growth of the fundamental instability component.

The total fluctuating velocity along the centerline of the jet is shown in Fig. 6 for the experiment and the corresponding computations are shown in Fig. 7. In each figure the excited case is compared to the unexcited case. The total fluctuation is composed of both the fundamental and the turbulent components. In the initial region of the jet, as a result of the amplification of the forced fundamental component, the velocity fluctuations for the excited case increase considerably as compared to the unexcited values. The fluctuations peak as the fundamental reaches its maximum. The magnitude of the peak is proportional to the magnitude of the fundamental's peak. Therefore, increasing the initial turbulence level reduces the peak of the fluctuation velocity in the excited case. As one moves downstream the fundamental decays and the fluctuating component is merely the turbulent component. By $x / 0=9$ the figures indicate that excitation has little effect on the downstream turbulent velocity. Also, comparing Fig. 7 to Fig. 6 indicates that the predicted effect of the initial turbulence on the total fluctuations along the jet centerline is overestimated as compared to the observed one. This is due to the overestimation of the amplification rates of the fundamental. However the theory predicts the same observed trend: increasing the initial level of turoulence decreases the peak of the total fluctuations

The development of the momentum thickness along the jet is shown in Figs. 8 and 9 . Figure 8 is for 0.5 percent initial turbulence while Fig. 9 is for 5 percent initial turbulence. Both theory and experiment indicate that excitation increases the momentum thickness of the jet. Comparing Fig. 9 to Fig. 8 indicates that the enhancement in the momentum thickness is reduced by increasing the initial level of the turbulence. Higher initial turbulence levels reduce the amplification of the fundamental and result in a lcwer growth rate of the jet. This observed behavior of the momentum thickness under excitation is also predicted by the theory as Figs, $8(b)$ and $g(b)$ indicate. However, further downstream the measured momentum thickness is higher than that calculated. As Fig. 5 has indicated. around $x / D=4$ a strong subharmonic of the fundamental is generated which results in further energy drain from the mean flow. This causes the observed higher values of the momentum thickness.

The mechanism of the jet growth is explained in Fig. 10. From Eq. (1) the growth rate of the jet is determined by the mean flow energy drain. This in turn is composed of two parts: Energy drained by the fundamental component, $|A| 2 \tilde{I}_{R S}$, and energy drained by the turbulent component, $E$ IRS. These two components as well as their sum are shown in Fig. 10. The figure shows that in the initial region of the jet the energy drain from the mean flow is enhanced due to the growth of the fundamental. Further downstream as the fundamental decays the growth rate is mainly due to the energy absorbed by the turbulence. As one increases the initial level of the turbulence ( $F i g .10(b)$ ), the amplification of the fundamental is reduced and therefore less energy is drained from the mean flow by the fundamental. The net result is therefore that higher initial turbulence level reduces the enhancement of the growth rate caused by the excitation.

\section{Conclusions}

The effect of initial core turbulence on the development of an excited jet was considered. Increasing the initial turbulence was found to reduce the amplification of the fundamental component and hence lowers the spreading rate of the excited jet. A comparison between the observations and the modified theory of Mankbadi \& Liub was made. The theory predicts the same qualitative behavior of the effect of initial turbulence. The theory does not include the generation of subharmonics which results in overestimating the amplification of the fundamental. 


\section{References}

1. Bradshaw, P., Ferriss, D.H., and Johnson, R.F., "Turbulence in the Noise-Producing Region of a Circular Jet," Journal of Fluid Mechanics. Vol. 19, Pt. 4, Aug. 1964, pp. 591-624ff.

2. Zaman, K.B.M.Q, and Hussain, A.K.M.F., "Turbulence Supression in Free Shear Flows by Controlled Excitation." Journal of Fluid Mechanics, Vol. 103, Feb. 1981, pp. 133-160.

3. Conen, J. and Wygnanski, I., "The Evolution of Instabilities in the Axisymmetric Jet. Part 2. The Flow Resulting From the Interaction Between Two Waves, Journal of Fluid Mechanics. Yo1. 176, Mar. 1987, pp. 221- 235.

4. Zaman, K.B.M.Q. and Hussain, A.K.M.F. "Vortex Pairing in a Circular Jet Under Controlled Excitation. Part 1. General Jet Response," Journal of Fluid Mechanics, Vol. 101, Pt. 3 , Dec. 11, 1980, pp. 449-491.

5. Chan, Y.Y. a'd Templin, J.T., "Suppression of Spatial Waves by Distortion of Jet Velocity Profile," Physics of Fluids, Vol. 17, No. 11, Nov. 1974, pp. 2124-2125
6. Mankbadi, R. and Liu, J.T.C., "A Study of the Interactions Between Large-Scale Coherent Structures and Fine-Grained Turbulence in a Round Jet." Philosophical Transactions of the Royal Society of London, Series A, Vol. 298, No. 1443, Jan. 21, 1981, pp. 541-602.

7. Raman, G., Zaman, K.B.M.Q., and Rice, E.J. "Initial Turbulence Effect on Jet Evolution With and Without Tonal Excitation." AIAA Paper 87-2725. Oct. 1987. (NASA TM-100178).

8. Michalke. A.. "Survey on Jet Instability Theory," Progress in Aerospace Sciences. Vol. 21, No. 3, 1984, pp. 159-199.

9. Mankbadi, R.R., "On the Interaction Between Fundamental and Subharmonic Instability Waves in a Turbulent Round Jet," Journal of Fluid Mechanics, Voi. 160. Nov. 1985, pp. 385-419. 

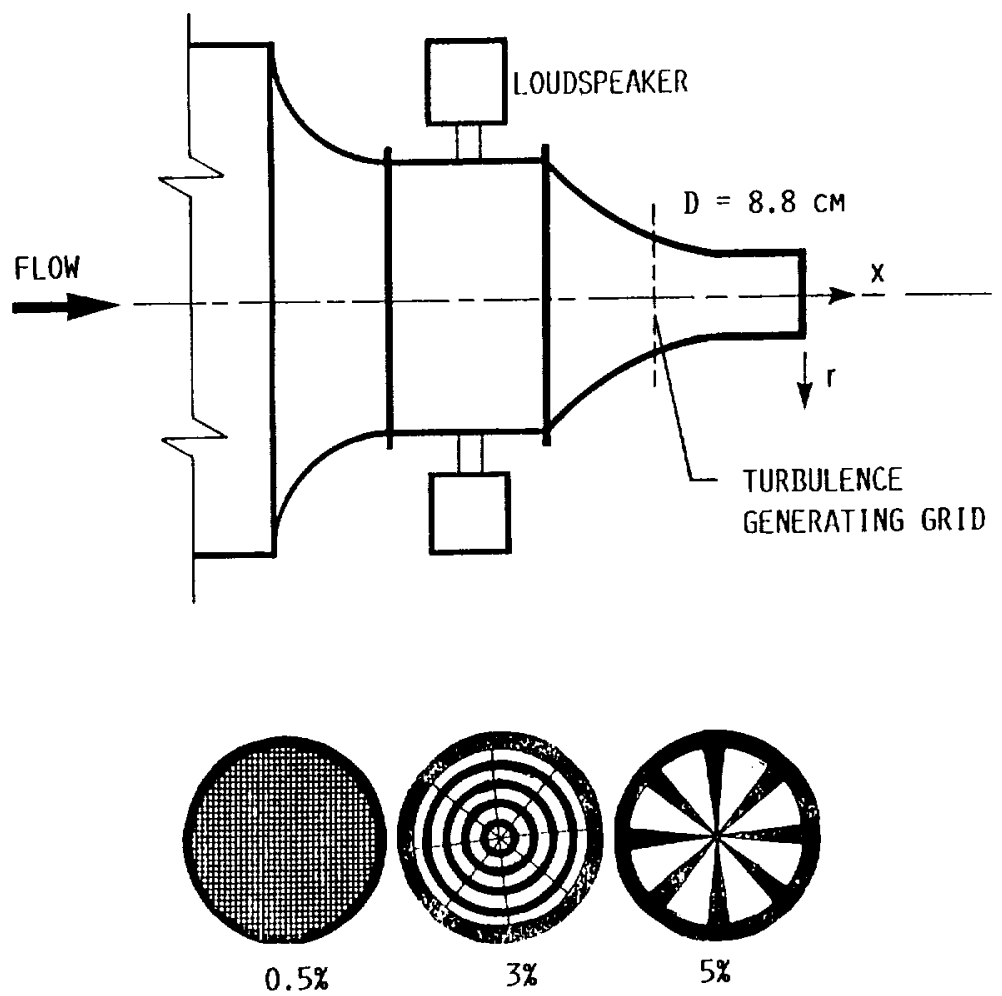

FIGURE 1. - SCHEMATIC OF JET FACILITY. THE THREE GRID CASES ARE IDENTIFIED BY THE SHOWN NOMINAL TURBULENCE INTENSITIES.

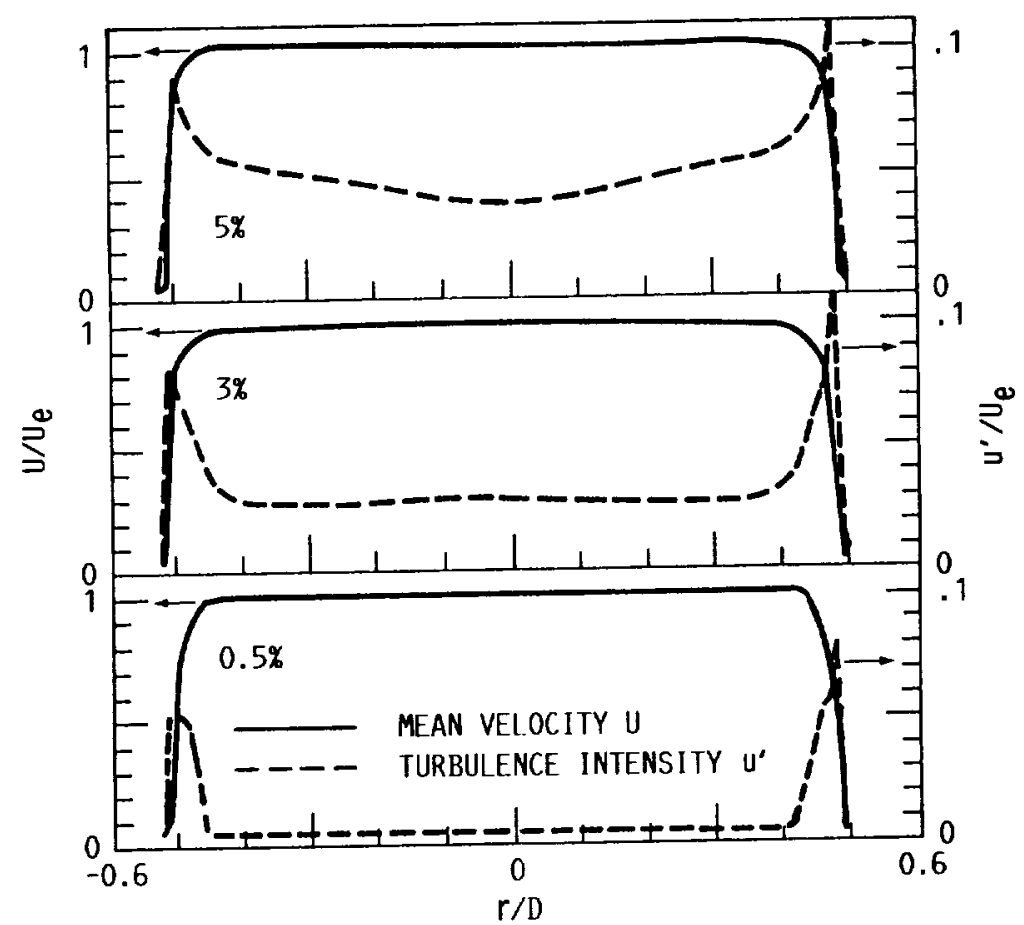

FIGURE 2. - RADIAL VARIATION OF MEAN VELOCITY AND THE TURBULENCE INTENSITY AT THE JET EXIT FOR THE THREE CASES INDICATED. 


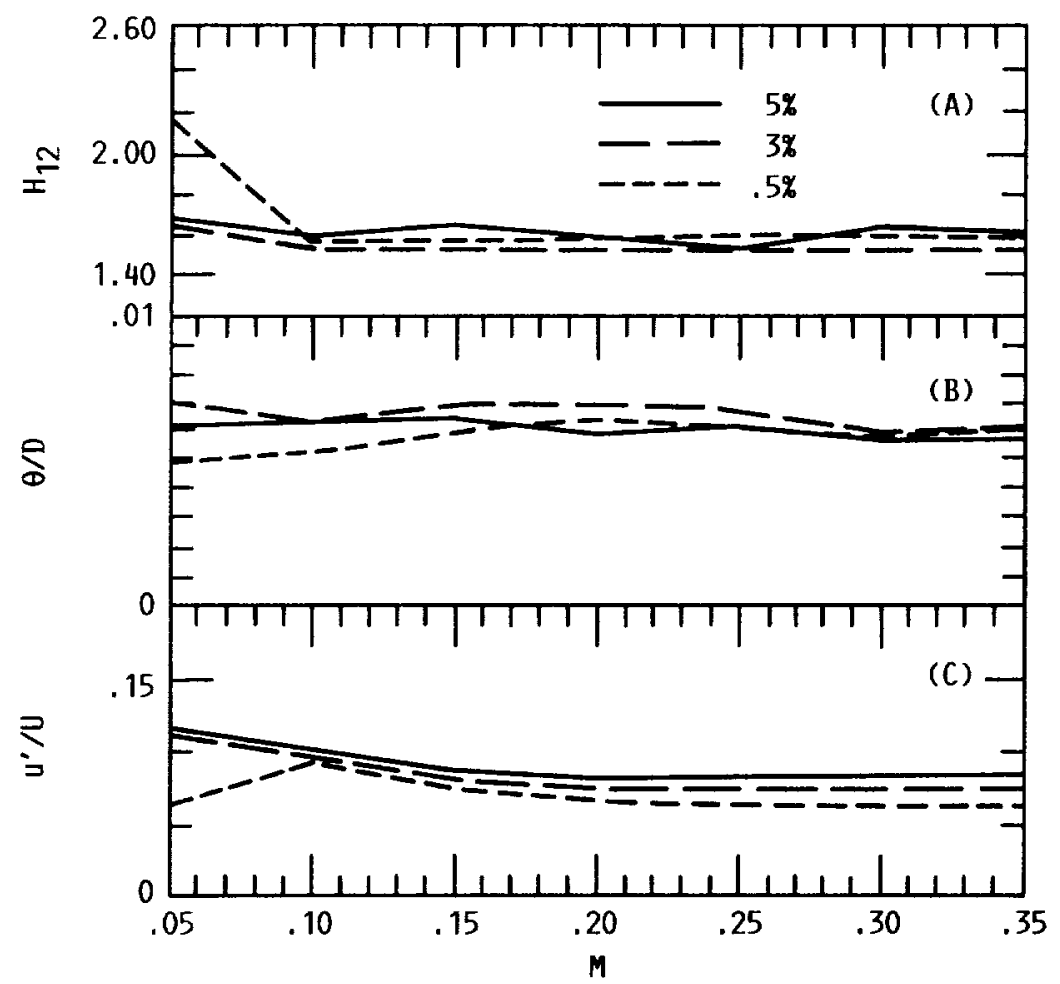

FIGURE 3. - BOUNDARY LAYER CHARACTERISTICS MEASURED 0.5 MM DOWNSTREAM OF THE JET EXIT. (A) SHAPE FACTOR, (B) MOMENTUM THICKNESS, (C) PEAK FLUCTUATION INTENSITY.

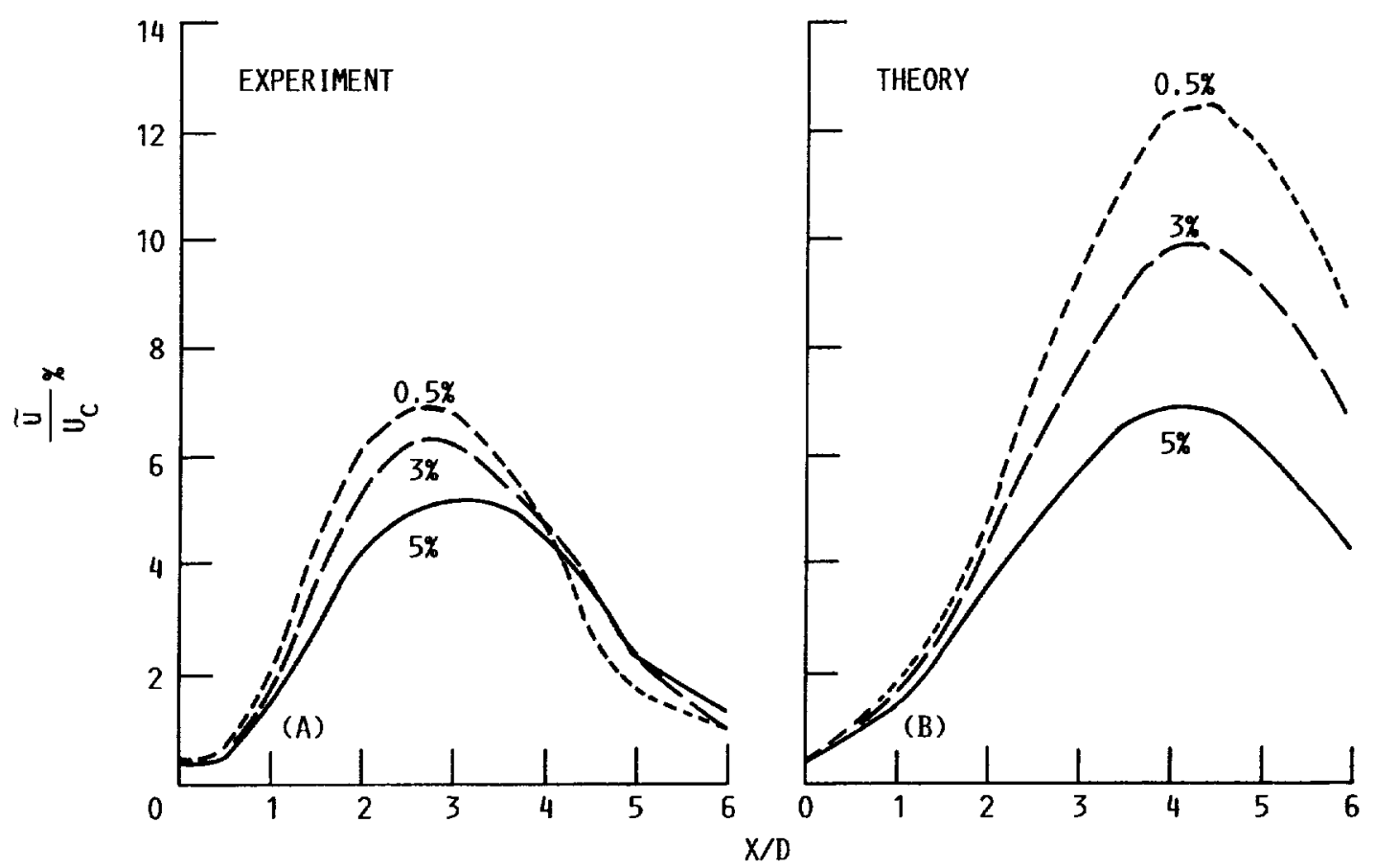

FIGURE 4. - DEVELOPMENT OF THE FUNDAMENTAL COMPONENT ALONG THE JET AT SEVERAL INITIAL LEVELS OF TURBULENCE. 


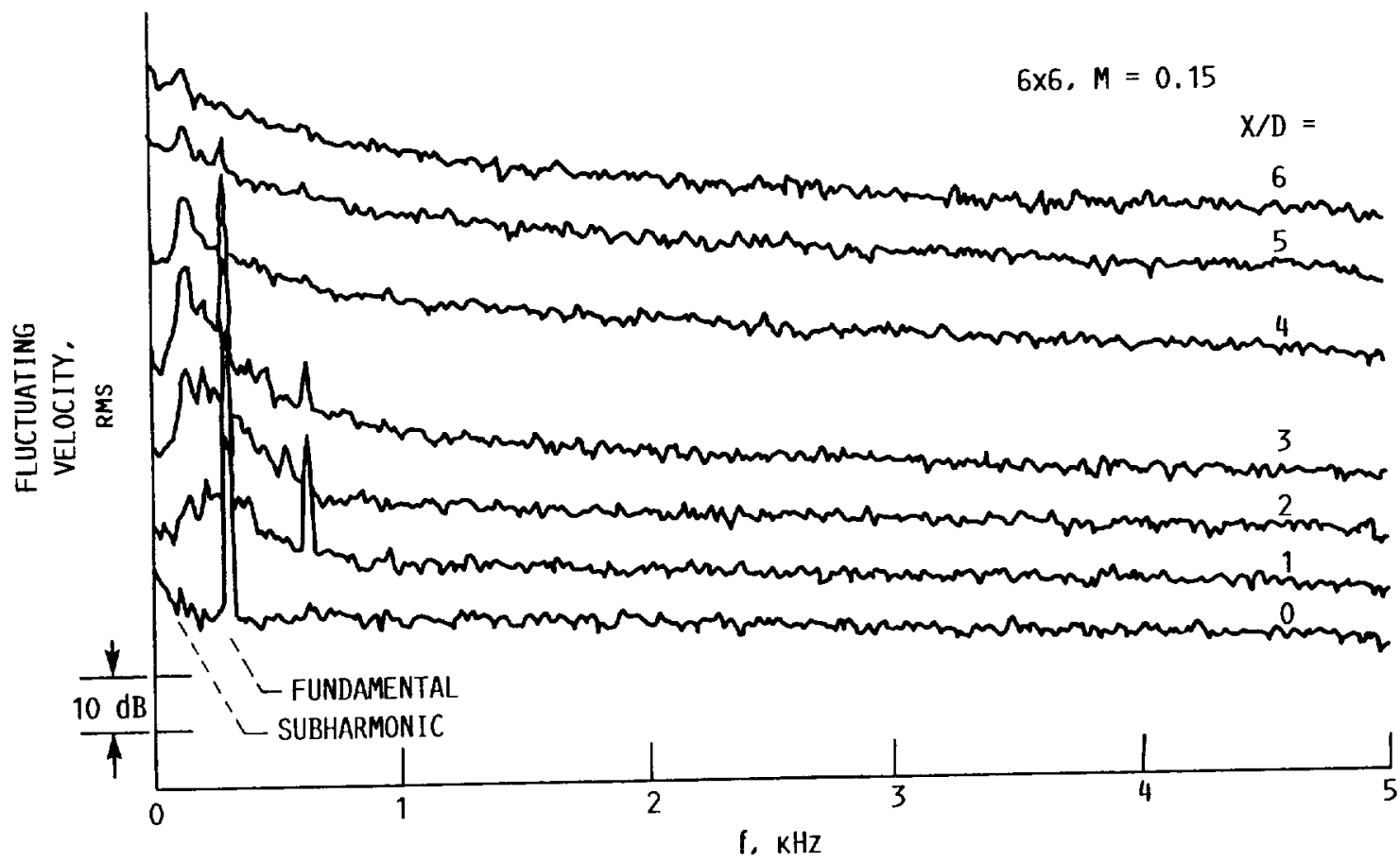

FIGURE 5. - U-SPECTRA MEASURED AT JET CENTERL INE FOR INITIAL LEVEL OF TURBULENCE $=0.5$ PERCENT. THE ORDINATE SPANS -70 TO $-40 \mathrm{~dB}$; BANDWIDTH $=0.5 \mathrm{~Hz}$.

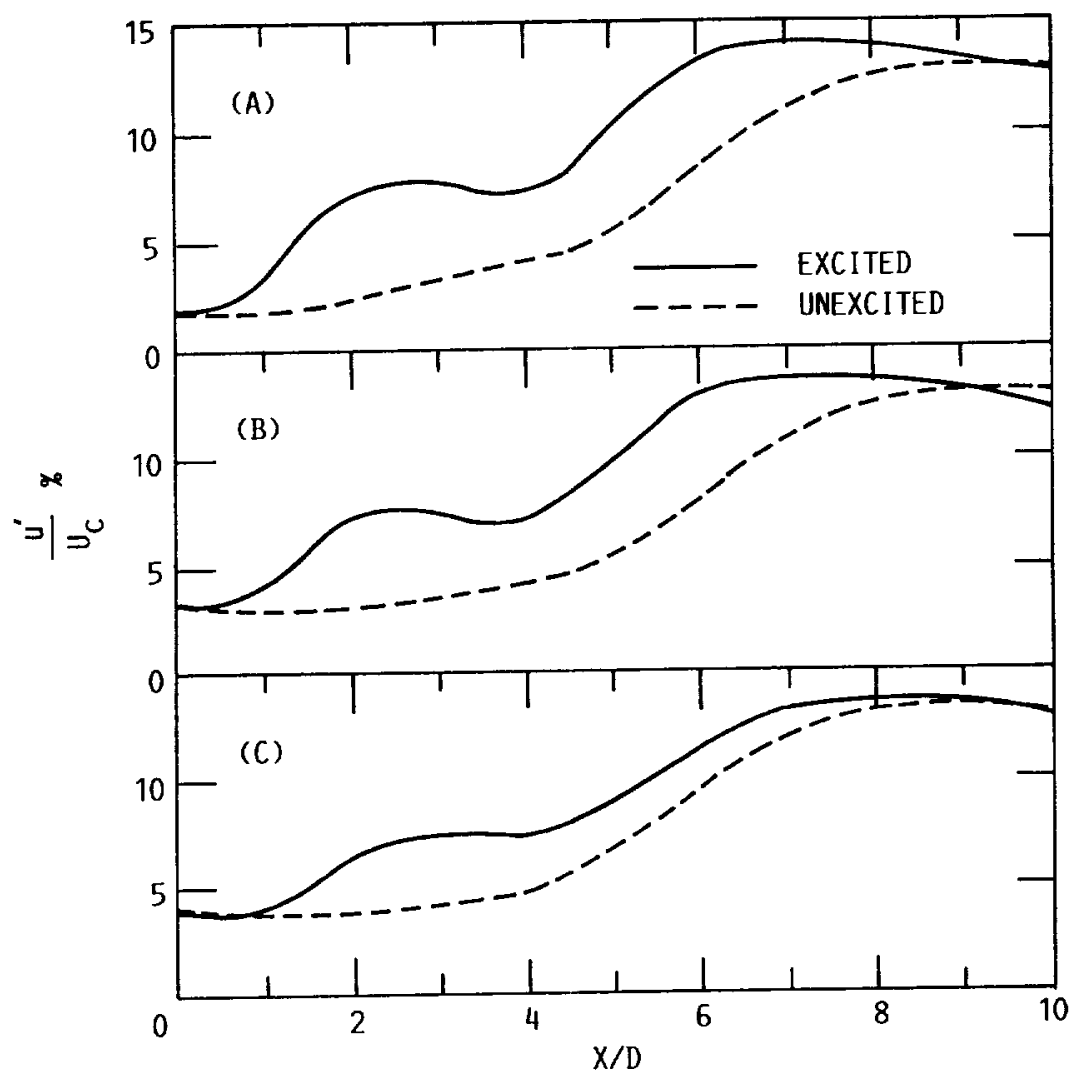

FIGURE 6. - MEASURED TOTAL VELOCITY FLUCTUATIONS ALONG THE JET CENTERLINE. INITIAL CORE TURBULENCE:

(A) 0.5 PERCENT, (B) 3 PERCENT, AND (C) 5 PERCENT. 


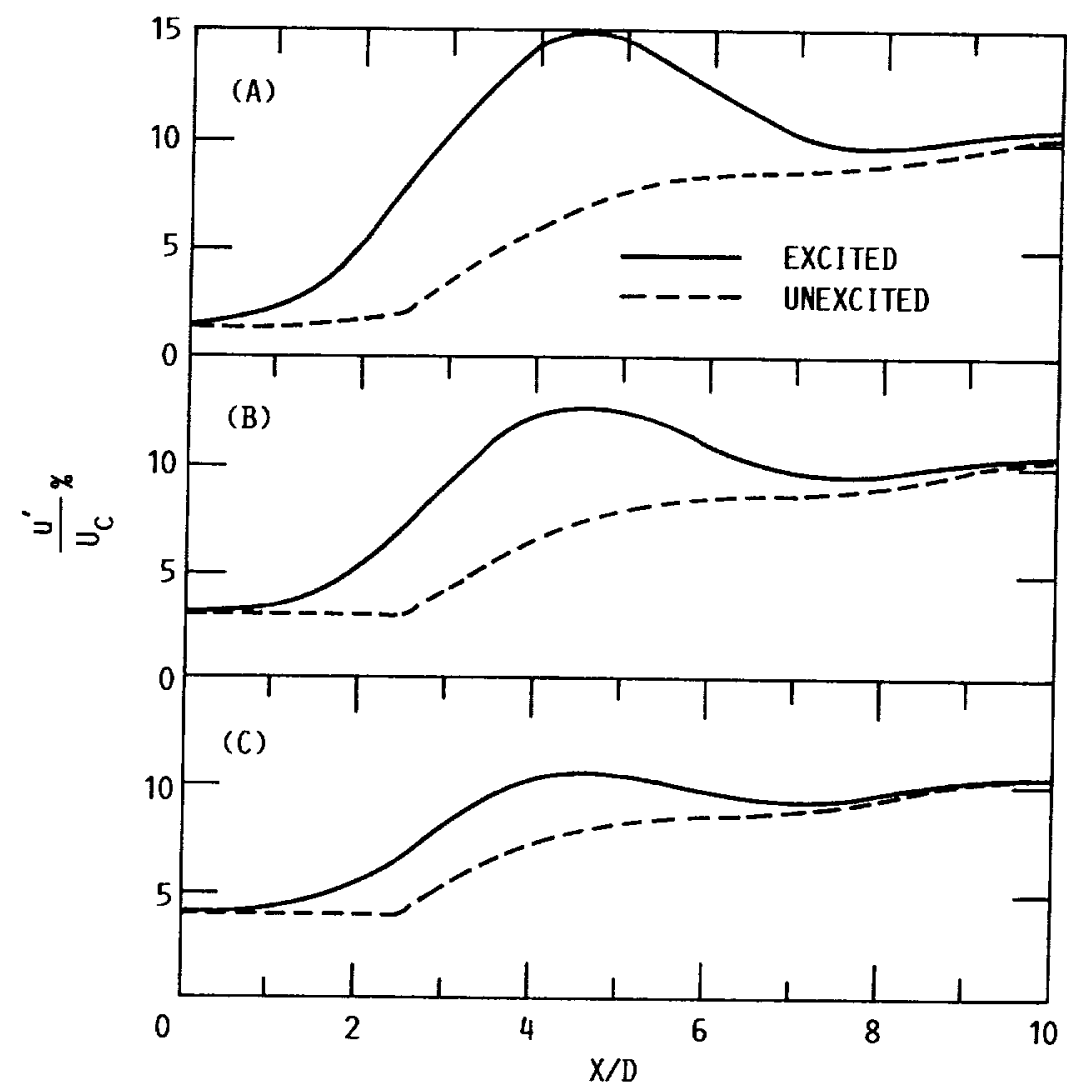

FIGURE 7. - CALCULATED TOTAL VELOCITY FLUCTUATIONS ALONG THE JET CENTERLINE. INITIAL CORE TURBULENCE : (A) 0.5 PERCENT, (B) 3 PERCENT AND (C) 5 PERCENT.

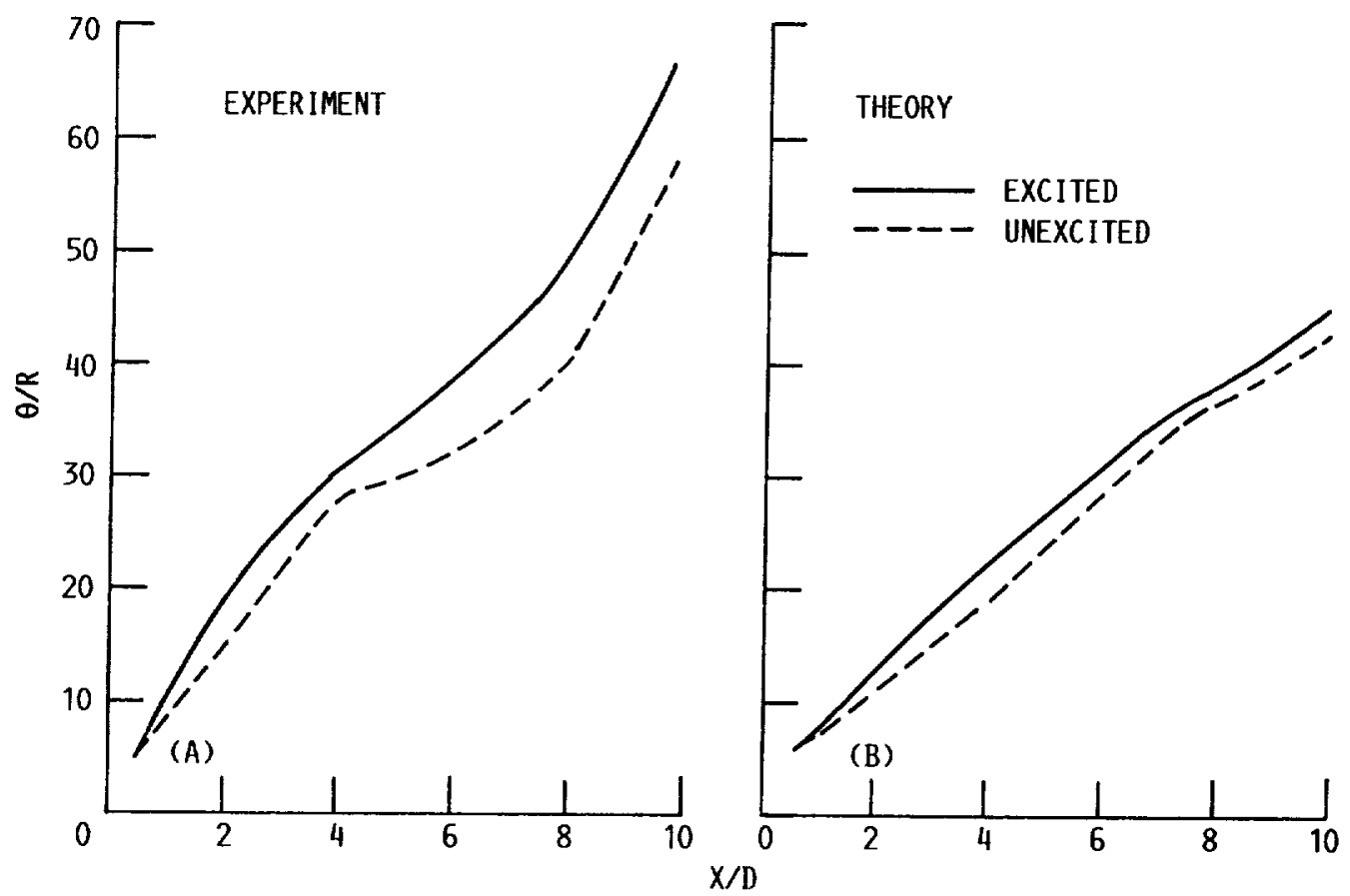

FIGURE 8. - DEVELOPMENT OF THE MOMENTUM THICKNESS ALONG THE JET AT INITIAL CORE TURBULENCE OF 0.5 PERCENT. 


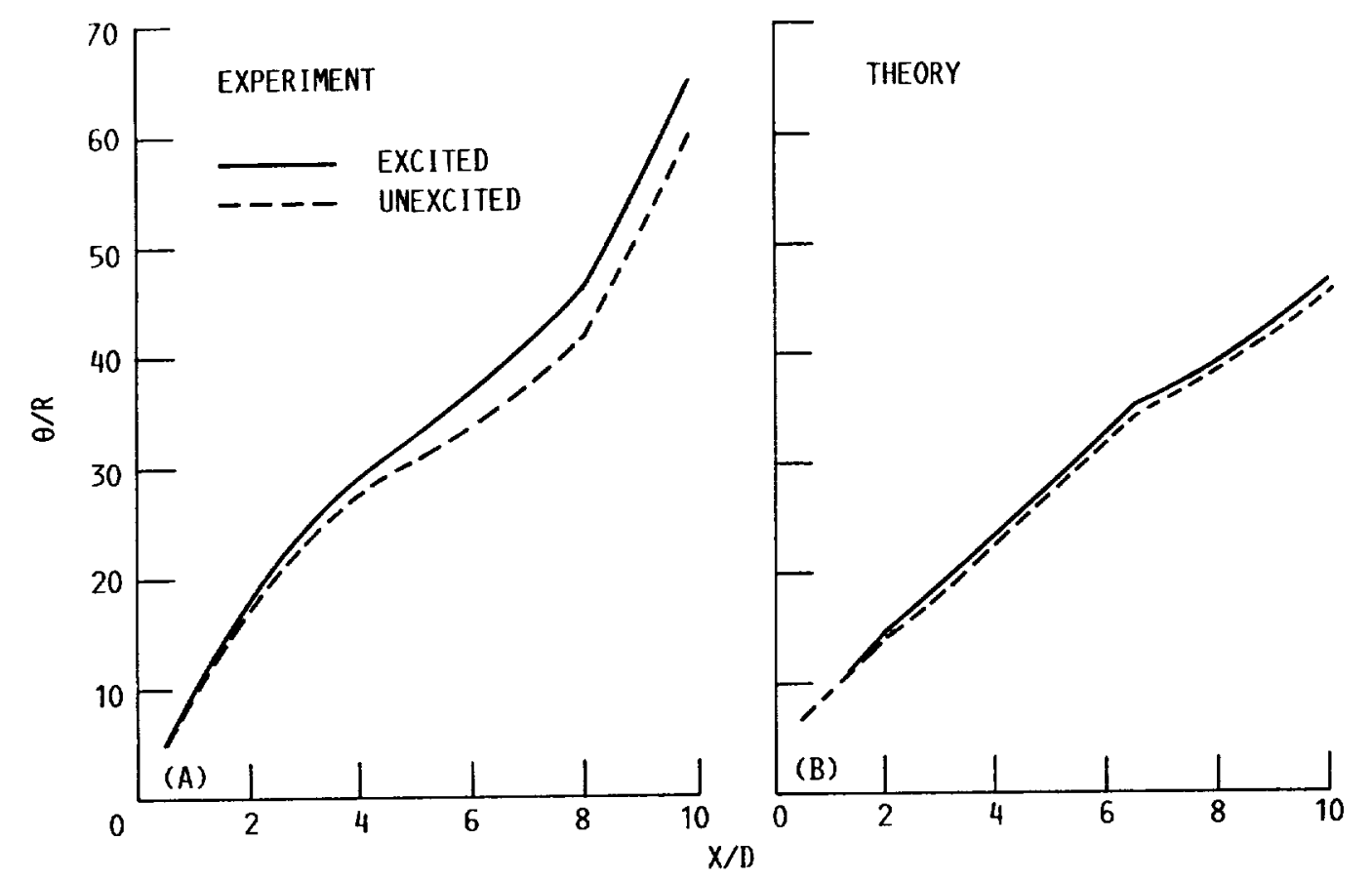

FIGURE 9. - DEVELOPEMENT OF THE MOMENTUM THICKNESS ALONG THE JET AT INITIAL CORE TURBULENCE OF 5 PERCENT.

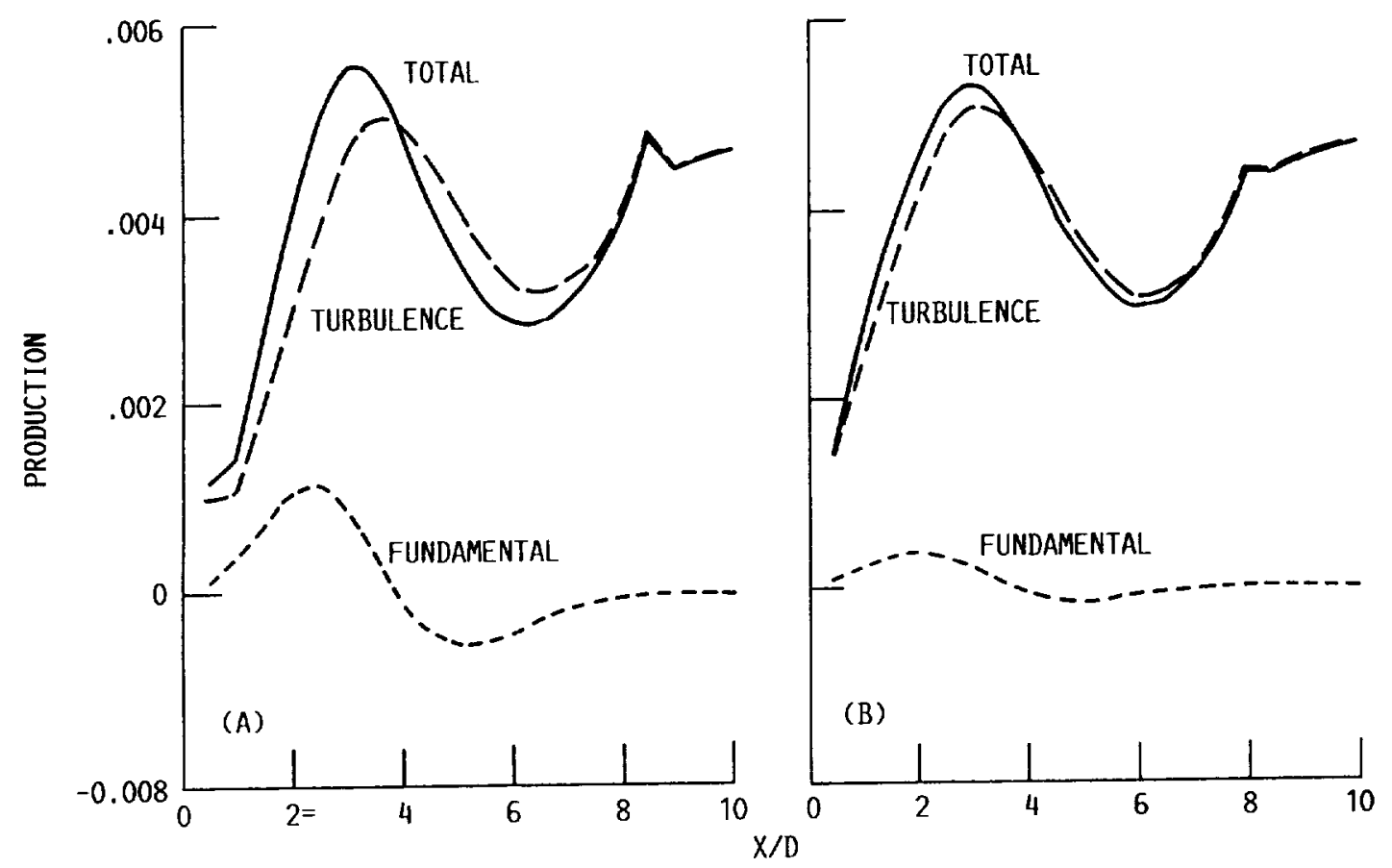

FIGURE 10. - MEAN FLOW ENERGY DRAIN BY THE FUNDAMENTAL AND THE TURBULENCE. INITIAL CORE TURBULENCE: (A) 0.5 PERCENT AND (B) 5 PERCENT. 


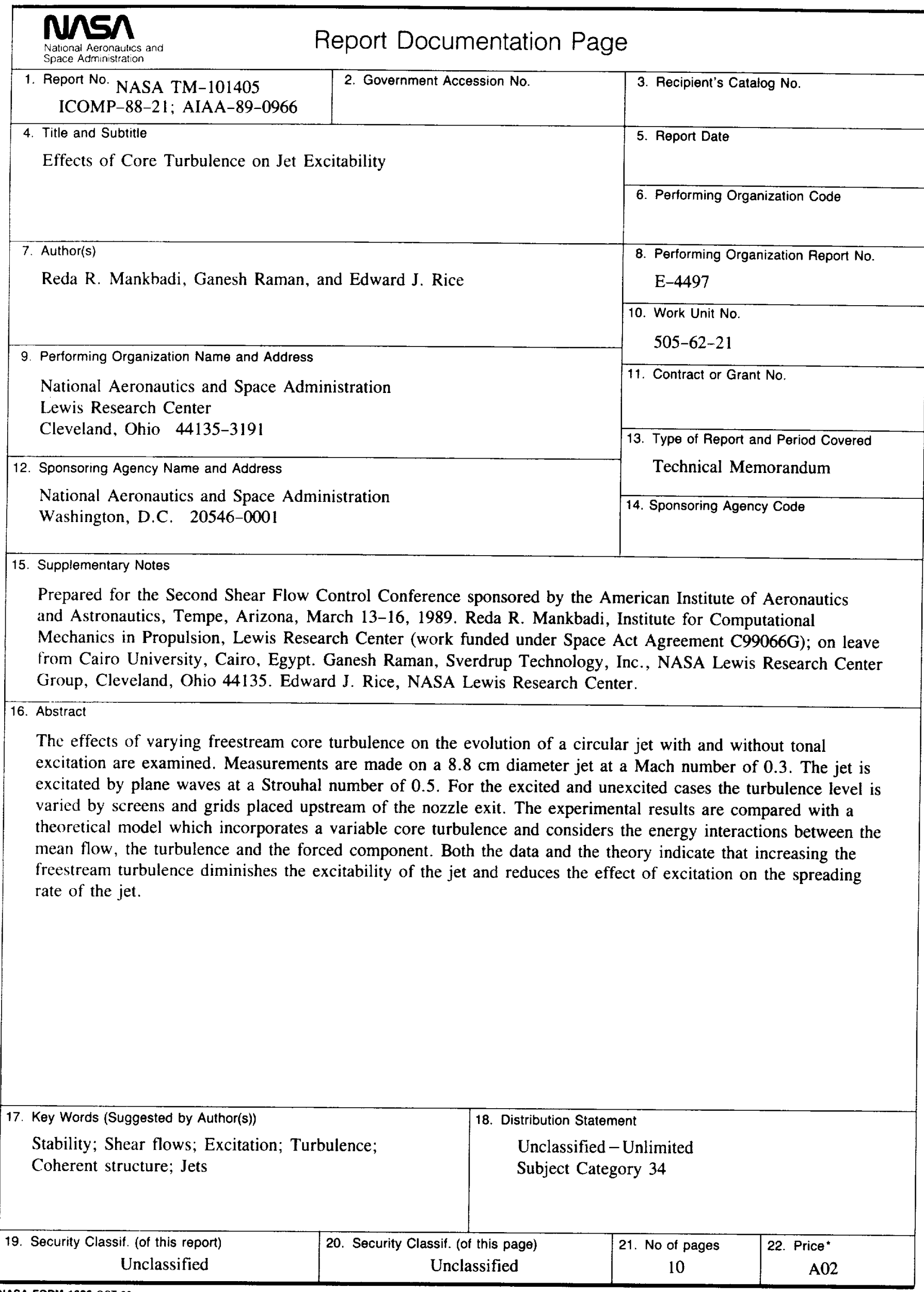

